\title{
THE CONSTITUTIVE EQUATIONS FOR RATE SENSITIVE PLASTIC MATERIALS*
}

\author{
BY \\ P. PERZYNA \\ Institute of Basic Technical Research, Polish Academy of Sciences
}

Summary. The principal aim of the present paper is to generalize the one-dimensional constitutive equations for rate-sensitive plastic materials to general states of stress. The dynamical yield conditions for elastic, visco-plastic materials are discussed and new relaxation functions are introduced. Solutions of the relaxation equations for such materials are given.

1. Introduction. For general types of stress and strain rate, possible constitutive equations were first given by Hohenemser and Prager [6]†; they can be written in the form (see Prager [9], [10])

$$
2 \eta \epsilon_{i i}^{\cdot P}=2 k\langle F\rangle \frac{\partial F}{\partial \sigma_{i i}},
$$

where

$$
F=\frac{J_{2}^{1 / 2}}{k}-1=\frac{\left\{s_{i j} s_{i j} / 2\right\}^{1 / 2}}{k}-1
$$

is the statical yield function and $s_{i j}$ denote components of the stress deviation and $\epsilon_{i j}^{P}$ the components of the plastic or, more generally, the anelastic strain tensor, $\eta$ is a coefficient of viscosity, and $k$ the yield stress in simple shear, $J_{2}$ represents the second invariant of the stress deviation, and the dot denotes differentiation with respect to time. The symbol $\langle F\rangle$ is defined as follows:

$$
\langle F\rangle= \begin{cases}0 & \text { for } F \leq 0 \\ F & \text { for } F>0 .\end{cases}
$$

Taking the elastic strains into consideration, and denoting by $e_{i j}$ the components of the strain deviation, and by $\mu$ and $K$ elastic constants, we obtain the relations

$$
\begin{array}{ll}
\dot{e_{i i}}=\frac{1}{2 \mu} s_{i i}+\frac{1-\dot{k J} J^{-1 / 2}}{2 \eta} s_{i i}, & \text { if } J_{2}^{1 / 2}>k, \\
\dot{e_{i i}}=\frac{1}{2 \mu} \dot{s}_{i i}, & \text { if } J_{2}^{1 / 2} \leq k, \\
\dot{\epsilon_{i i}}=\frac{1}{3 K} \sigma_{i i} &
\end{array}
$$

which were studied by Freudenthal [5].

*Received May 14, 1962. The paper was written while the author was a Visiting Research Associate in Applied Mathematics at Brown University.

†Numbers in square brackets refer to the Bibliography at the end of the paper. 
2. New constitutive equations. To generalize (1), let us replace $2 k\langle F\rangle$ by $\gamma^{0} \Phi(F)$, where $\gamma^{0}$ denotes a physical constant of the material, $F$ is again defined by (2), and the function $\Phi(F)$ satisfies the conditions

$$
\left.\begin{array}{lll}
\Phi(F)=0 & \text { if } & F \leq 0 \\
\Phi(F) \neq 0 & \text { if } & F>0
\end{array}\right\}
$$

When elastic strains are added, the following equivalent forms of the constitutive equation are obtained

$$
\begin{array}{ll}
\dot{e}_{i j}=\frac{1}{2 \mu} s_{i j}+\gamma^{0} \Phi\left(H^{\prime}\right) \frac{\partial F}{\partial \sigma_{i j}}, & \text { for } F>0 \\
\dot{e}_{i j}=\frac{1}{2 \mu} s_{i j}, & \text { for } F \leq 0 \\
\epsilon_{i i}=\frac{1}{3 K} \sigma_{i i}, &
\end{array}
$$

or

$$
\begin{aligned}
& e_{i j}^{\cdot}=\frac{1}{2 \mu} s_{i j}^{\cdot}+\gamma \Phi\left(F^{\prime}\right) \frac{s_{i j}}{J_{2}^{1 / 2}}, \quad \text { for } \quad J_{2}^{1 / 2}>k, \\
& e_{i j}=\frac{1}{2 \mu} s_{i j}, \\
& \text { for } \left.J_{2}^{1 / 2} \leq k,\right\} \\
& \dot{\epsilon}_{i i}=\frac{1}{3 K} \sigma_{i i},
\end{aligned}
$$

where $\gamma=\gamma^{0} / 2 k$.

The function $\Phi(F)$ may be chosen to represent the results of tests on the behavior of metals under dynamic loading.

The general assumptions in Eqs. (7) are the same as those introduced by Malvern in his nonlinear relations for one-dimensional problems [7]. The relations (7) involve the assumption that the rate of increase of the plastic components of the strain tensor be a function of the excess stresses above the static yield condition. The elastic components of the strain tensor are considered to be independent of the strain rates. In Eq. (7) the strain hardening effect is neglected. All available experimental data indicates that strain-hardening of dynamically strained mild steel is very small (see, for instance, Campbell's recent investigations [2] and [3] concerning the behavior of mild steel under dynamic loading).

Consider now the anelastic part of the relations (7)

$$
\epsilon_{i i}^{\cdot P}=\gamma \Phi(F) \frac{s_{i i}}{J_{2}^{1 / 2}} .
$$

Squaring both sides of Eq. (8) and denoting by $I_{2}^{P}=\frac{1}{2} \epsilon_{i i}^{P} \cdot \dot{\epsilon}_{i j}^{P}$ the second invariant of an elastic strain rate tensor, we obtain

$$
\left(I_{2}^{P}\right)^{1 / 2}=\gamma \Phi(F)
$$

By (9), we can write

$$
J_{2}^{1 / 2}=k\left[1+\Phi^{-1}\left(\left[I_{2}^{P}\right]^{1 / 2} / \gamma\right)\right]
$$






Fia. 1.

where $\Phi^{-1}$ denotes the inverse function of $\Phi$, (Fig. 1). It can be seen from (10) that the constitutive equations (7) lead to a similar result as the theory of plasticity, for isotropic work hardening material. But in the theory of plasticity for isotropic work-hardening, the radius $R$ of the cylindrical yield locus in stress space depends on the strain, whereas in the case considered here $R$ depends on strain rate according to

$$
R=R_{0}\left[1+\Phi^{-1}\left(\left[I_{2}^{P}\right]^{1 / 2} / \gamma\right)\right],
$$

where $R_{0}=2^{1 / 2} k$ denotes the radius of the statical yield cylinder (Fig. 2).

In the theory of plasticity for isotropic work-hardening material we have three possibilities according to whether $J_{2}^{*}>0$ (loading), $J_{2}^{*}=0$ (neutral loading), or $J_{2}^{*}<0$ (unloading).

In visco-plasticity, since $J_{2}$ is a function of the strain rate, the plastic flow (relaxation effect) occurs if $J_{2}>k^{2}$, independent of whether $J_{2}^{*}>0, J_{2}^{*}=0$ or $J_{2}^{*}<0$.

The condition

gives the relation

$$
s_{i j} e_{i j}=\frac{1}{2 \mu} J_{2}^{\cdot}+2 \gamma \Phi(F) J_{2}^{1 / 2}>0
$$

$$
4 \mu \gamma \Phi\left(F^{\prime}\right) J_{2}^{1 / 2}>\left|J_{2}^{*}\right| .
$$

Due to the relations (7), the material will be elastic on the path $O P_{0}$, but on the path $P_{0} P_{1} P_{1}^{\prime} P_{2}$ it will be elastic, visco-plastic, (Fig. 2).

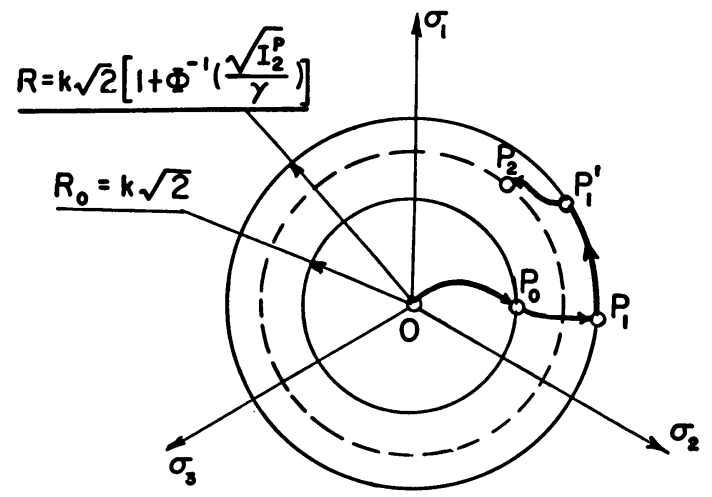

Fig. 2. 
3. Relaxation process. To discuss a relaxation process for general states of stress, let us consider an elastic, visco-plastic body occupying the three dimensional region $V$ with the regular surface $S$ and investigate the following boundary value problem. Consider first the loading process in which the surface tractions $T_{i}$ are prescribed on the part $S_{1}$ of $S$ and vanish on the remainder $S_{2}$. This loading is to be followed by a relaxation process in which the surface velocities $v_{i}$ vanish on $S_{1}$, while the surface tractions $T_{i}$ continue to vanish on $S_{2}$. In a relaxation test that is to furnish useful information about the constitutive equations, the states of stress and strain must be homogeneous. A relaxation process defined in this manner we shall call an $A$-process.

Let us consider the tensor $\Gamma_{i}$ defined by the formula

$$
\Gamma_{i i}=\frac{1}{2} \int\left(T_{i} v_{i}+T_{i} v_{i}\right) d S .
$$

During an $A$-process the tensor $\Gamma_{i j}$ vanishes. Thus,

$$
\begin{aligned}
\Gamma_{i i} & =\frac{1}{2} \int\left(T_{i} v_{i}+T_{j} v_{i}\right) d S=\frac{1}{2} \int\left(\sigma_{i k} n_{k} v_{i}+\sigma_{i k} n_{k} v_{i}\right) d S \\
& =\frac{1}{2} \int \partial_{k}\left(\sigma_{i k} v_{i}+\sigma_{i k} v_{i}\right) d V=\frac{1}{2} \int\left(\sigma_{i k} \partial_{k} v_{i}+\sigma_{j k} \partial_{k} v_{i}\right) d V \\
& =\frac{1}{2} \int\left(\sigma_{i k} \epsilon_{k i}+\sigma_{i k} \epsilon_{k i}\right) d V=0 .
\end{aligned}
$$

The states of stress and strain are homogeneous during this type of relaxation test; we therefore have

$$
\frac{1}{2}\left(\sigma_{i k} \dot{\epsilon_{k i}}+\sigma_{i k} \dot{\epsilon_{k i}}\right)=0 .
$$

These conditions enable us to determine the state of stress as a function of time during a relaxation process.

Assume now that a certain state of stress characterized by $\sigma_{i j}^{(0)}$ (or $s_{i j}^{(0)}$ and $\sigma_{k k}^{(0)}$ ) and $J_{2}^{(0)}=s_{i j}^{(0)} s_{i j}^{(0)} / 2>k^{2}$ has been reached at the time $t=0$ and next the relaxation process occurs. Then by (7) and (16) we obtain a system of six differential equations with respect to $s_{i j}$ and $\sigma_{k k}$

$$
\begin{aligned}
\frac{1}{2}\left\{\left(s_{i k}+\frac{1}{3} \sigma_{l l} \delta_{i k}\right)\right. & {\left[\frac{1}{2 \mu} s_{k i}+\frac{1}{9 K} \sigma_{i l} \delta_{k i}+\gamma \Phi(F) \frac{s_{k i}}{J_{2}^{1 / 2}}\right] } \\
& \left.+\left(s_{j k}+\frac{1}{3} \sigma_{l l} \delta_{j k}\right)\left[\frac{1}{2 \mu} s_{k i}+\frac{1}{9 K} \sigma_{i l} \delta_{k i}+\gamma \Phi(F) \frac{s_{k i}}{J_{2}^{1 / 2}}\right]\right\}=0
\end{aligned}
$$

By assuming in (16) $i=j$ we have the condition

$$
\sigma_{i j} \epsilon_{i j}=0 \text {. }
$$

Multiplying Eq. (16) by $\sigma_{i i}$ we obtain

$$
\frac{1}{2}\left(\sigma_{i i} \dot{\epsilon_{k i}}+\sigma_{j i} \dot{\epsilon} \dot{\epsilon_{i}}\right) \sigma_{i j}=\sigma_{i i} \sigma_{i k} \dot{\epsilon_{k i}}=0 .
$$

Consider now the simpler boundary value problem in which the loading process is characterized by the surface tractions $T_{i}$ being prescribed on the entire surface $S$, while during a relaxation process the surface velocities $v_{i}$ vanish on the entire surface $S$. This kind of relaxation process we shall call a $B$-process. 
Because the states of stress and strain are homogeneous during a relaxation test, we now have conditions

$$
\epsilon_{i i}=0 .
$$

From (7) and (20) we obtain a system of five differential equations with respect to $s_{i j}$ in the form

$$
s_{i j}+2 \mu \gamma \Phi(F) \frac{s_{i j}}{J_{2}^{1 / 2}}=0 .
$$

The conditions (20) lead to two useful scalar conditions

$$
\begin{aligned}
s_{i j} \dot{e}_{i j} & =0, \\
s_{i j} s_{i k} \dot{e}_{k i} & =0 .
\end{aligned}
$$

It is worth noting that these conditions are valid for $A$-processes in incompressible materials because then

$$
\epsilon_{i i}=0 \text {. }
$$

The validity of (22) and (23) for an $A$-process under condition (24) follows readily from (18) and (19).

According to (12) and (22) during $A$-processes in incompressible materials and during $B$-processes in any materials we have a relaxation equation for $J_{2}$ in the following form:

$$
J_{2}^{\cdot}+4 \mu \gamma \Phi\left(\frac{J_{2}^{1 / 2}}{k}-1\right) J_{2}^{1 / 2}=0 .
$$

This can be written as a nonlinear Volterra integral equation of the second kind

$$
J_{2}=J_{2}^{(0)}-4 \mu \gamma \int_{0}^{t} J_{2}^{1 / 2} \Phi\left(\frac{J_{2}^{1 / 2}}{k}-1\right) d t .
$$

Under the assumption that the integrand $J_{2}^{1 / 2} \Phi\left(J_{2}^{1 / 2} / k-1\right)$ satisfies a Lipschitz condition, that is that

$$
\left|\left(J_{2}^{\prime}\right)^{1 / 2} \Phi\left(\frac{\left(J_{2}^{\prime}\right)^{1 / 2}}{k}-1\right)-\left(J_{2}^{\prime \prime}\right)^{1 / 2} \Phi\left(\frac{\left(J_{2}^{\prime \prime}\right)^{1 / 2}}{k}-1\right)\right|<N_{0}\left|J_{2}^{\prime}-J_{2}^{\prime \prime}\right|,
$$

where $N_{0}$ is a positive constant, the solution of Eq. (26) can be obtained by the iteration method based on recurrence formula

$$
J_{2}^{(n+1)}=J_{2}^{(0)}-4 \mu \gamma \int_{0}^{t}\left(J_{2}^{(n)}\right)^{1 / 2} \Phi\left(\frac{\left(J_{2}^{(n)}\right)^{1 / 2}}{k}-1\right) d t .
$$

It is easily verified that the series

$$
J_{2}^{(0)}+\sum_{n=0}^{\infty}\left[J_{2}^{(n+1)}(t)-J_{2}^{(n)}(t)\right]
$$

is absolutely and uniformly convergent, and its sum

$$
J_{2}(t)=\lim _{n \rightarrow \infty} J_{2}^{(n)}(t)
$$

is the solution of the integral equation (26), and hence of the differential equation (25). Of course, the solution (30) is valid only in the nonelastic region $J_{2}>k^{2}$. 
4. Generalization. It is obvious that the constitutive equations (6) are valid for any function $F$ representing the statical yield condition provided the assumptions concerning the perfectly plastic materials are satisfied, i.e. that the function $F$ does not depend on the strains. For instance, for

$$
F=\frac{f\left(J_{2}, J_{3}\right)}{c}-1,
$$

where $J_{3}$ is the third invariant of the stress deviation, and $c$ is a constant, the equations (6) have the form

$$
\begin{array}{rlrl}
\dot{e}_{i i} & =\frac{1}{2 \mu} s_{i j}+\frac{\gamma^{0}}{c} \Phi\left[\frac{f\left(J_{2}, J_{3}\right)}{c}-1\right] \frac{\partial f}{\partial \sigma_{i j}}, & \text { if } & f\left(J_{2}, J_{3}\right)>c, \\
\dot{e_{i j}} & =\frac{1}{2 \mu} s_{i j}, & \text { if } f\left(J_{2}, J_{3}\right) \leq c, \\
\epsilon_{i i}=\frac{1}{3 K} \sigma_{i i} . &
\end{array}
$$

Considering the nonelastic part of the strain rate defined by (32) we have

$$
\epsilon_{i i}^{\cdot P}=\frac{\gamma^{0}}{c} \Phi\left[\frac{f\left(J_{2}, J_{3}\right)}{c}-1\right] \frac{\partial f}{\partial \sigma_{i j}} .
$$

Squaring both sides of Eq. (33) we obtain

$$
\left(I_{2}^{P}\right)^{1 / 2}=\frac{\gamma^{0}}{c} \Phi\left[\frac{f\left(J_{2}, J_{3}\right)}{c}-1\right]\left(\frac{\partial f}{\partial \sigma_{i i}} \frac{\partial f}{\partial \sigma_{i j}}\right)^{1 / 2} .
$$

According to (34) we have

$$
f\left(J_{2}, J_{3}\right)=\left\{1+\Phi^{-1}\left[\frac{c}{\gamma^{0}}\left(I_{2}^{P}\right)^{1 / 2}\left(\frac{\partial f}{\partial \sigma_{i j}} \frac{\partial f}{\partial \sigma_{i j}}\right)^{-1 / 2}\right]\right\} .
$$

This expression implicitly represents the dynamical yield condition for elastic, viscoplastic materials, and describes the dependence of the yield condition on the strain rate.

If we multiply the first equation (32) by $s_{i i}$ and use the identity

$$
\frac{\partial f}{\partial \sigma_{i i}}=\frac{\partial f}{\partial J_{2}} s_{i j}+\frac{\partial f}{\partial J_{3}} t_{i j}
$$

where $t_{i j}=s_{i k} s_{k j}-\frac{2}{3} J_{2} \delta_{i j}$, we have

$$
s_{i j} \dot{e}_{i j}=\frac{1}{2 \mu} J_{2}+2 \frac{\gamma^{0}}{c} \Phi(F)\left[\frac{\partial f}{\partial J_{2}} J_{2}+\frac{3}{2} \frac{\partial f}{\partial J_{3}} J_{3}\right] .
$$

Similarly, multiplying the first equation (32) by $s_{i k} s_{k i}$ we find

$$
s_{i k} s_{k i} e_{i j}=\frac{1}{2 \mu} J_{3}^{*}+3 \frac{\gamma^{0}}{c} \Phi(F)\left[\frac{\partial f}{\partial J_{2}} J_{3}+\frac{\partial f}{\partial J_{3}} g\left(J_{2}, J_{3}\right)\right]
$$

where $g\left(J_{2}, J_{3}\right)$ is a known function.

Consider now a relaxation process for the material characterized by the constitutive equations (32). Assume that during loading process a certain state of stress $\sigma_{i j}^{(0)}$ has 
been reached at the time $t=0$, and that then an $A$-process occurs. From (16) and (32) we obtain the following system of six differential equations for $s_{i i}$ and $\sigma_{i i}$ :

$$
\begin{aligned}
& \frac{1}{2}\left\{\left(s_{i k}+\frac{1}{3} \sigma_{l l} \delta_{i k}\right)\left[\frac{1}{2 \mu} s_{k i}+\frac{1}{9 K} \sigma_{i l} \delta_{k i}+\frac{\gamma^{0}}{c} \Phi\left(\frac{f}{c}-1\right) \frac{\partial f}{\partial \sigma_{i j}}\right]\right. \\
& \left.+\left(s_{i k}+\frac{1}{3} \sigma_{l l} \delta_{j k}\right)\left[\frac{1}{2 \mu} s_{k i}+\frac{1}{9 K} \sigma_{i l} \delta_{k i}+\frac{\gamma^{0}}{c} \Phi\left(\frac{f}{c}-1\right) \frac{\partial f}{\partial \sigma_{k i}}\right]\right\}=0 .
\end{aligned}
$$

During a $B$-process, (20) and (32) furnish the following system of five differential equations for $s_{i j}$ :

$$
s_{i j}+\frac{2 \mu \gamma^{0}}{c} \Phi\left[\frac{f\left(J_{2}, J_{3}\right)}{c}-1\right] \frac{\partial f}{\partial \sigma_{i j}}=0 .
$$

During a $B$-process or during an $A$-process for an incompressible material it is useful to study the change of the invariants $J_{2}$ and $J_{3}$.

By conditions (22) and (23) and by (37) and (38) we have

$$
\left.\begin{array}{r}
J_{2}^{*}+4 \mu \frac{\gamma^{0}}{c} \Phi(F)\left[\frac{\partial f}{\partial J_{2}} J_{2}+\frac{3}{2} \frac{\partial f}{\partial J_{3}} J_{3}\right]=0, \\
J_{3}^{*}+6 \mu \frac{\gamma^{0}}{c} \Phi(F)\left[\frac{\partial f}{\partial J_{2}} J_{3}+\frac{\partial f}{\partial J_{3}} g\left(J_{2}, J_{3}\right)\right]=0 .
\end{array}\right\}
$$

Introducing the notation

$$
\left.\begin{array}{l}
\beta_{1}\left(J_{2}, J_{3}\right)=4 \mu \frac{\gamma^{0}}{c} \Phi\left(F^{\prime}\right)\left[\frac{\partial f}{\partial J_{2}} J_{2}+\frac{3}{2} \frac{\partial f}{\partial J_{3}} J_{3}\right], \\
\beta_{2}\left(J_{2}, J_{3}\right)=6 \mu \frac{\gamma^{0}}{c} \Phi\left(F^{\prime}\right)\left[\frac{\partial f}{\partial J_{2}} J_{3}+\frac{\partial f}{\partial J_{3}} g\left(J_{2}, J_{3}\right)\right],
\end{array}\right\}
$$

and assuming that the functions $\beta_{1}$ and $\beta_{2}$ satisfy the Lipschitz inequalities, we write

$$
\left.\begin{array}{l}
J_{2}^{(n+1)}=J_{2}^{(0)}-\int_{0}^{t} \beta_{1}\left[J_{2}^{(n)}(\xi), J_{3}^{(n)}(\xi)\right] d \xi, \\
J_{3}^{(n+1)}=J_{3}^{(0)}-\int_{0}^{t} \beta_{2}\left[J_{2}^{(n)}(\xi), J_{3}^{(n)}(\xi)\right] d \xi .
\end{array}\right\}
$$

The change of the function $f$ during a relaxation process of type $B$ or of type $A$ for an incompressible material is determined by the relations

$$
\begin{aligned}
f^{\cdot} & =\frac{\partial f}{\partial J_{2}} J_{2}^{\cdot}+\frac{\partial f}{\partial J_{3}} J_{3}^{\cdot}, \\
f\left(J_{2}, J_{3}\right) & =\lim _{n \rightarrow \infty} f\left[J_{2}^{(n)}, J_{3}^{(n)}\right] .
\end{aligned}
$$

5. Special types of the function $\Phi$. Let us assume that the function $\Phi(F)$ has the following form

$$
\Phi(F)=F^{\delta}=\left(\frac{J_{2}^{1 / 2}}{k}-1\right)^{\delta}
$$


Then the stress-strain relations (7) can be written as

$$
\begin{aligned}
& \dot{e}_{i i}=\frac{1}{2 \mu} s_{i i}+\gamma\left(\frac{J_{2}^{1 / 2}}{k}-1\right)^{\delta} \frac{s_{i j}}{J_{2}^{1 / 2}}, \quad \text { if } \quad J_{2}^{1 / 2}>k, \\
& e_{i i}^{\cdot}=\frac{1}{2 \mu} s_{i j} \\
& \text { if } \left.\quad J_{2}^{1 / 2} \leq k,\right\}
\end{aligned}
$$

When the elastic deformations are negligible in comparison with the plastic deformations, then for one-dimensional states of stress we obtain, by (47), the relation

$$
\epsilon=\gamma\left(\frac{\sigma}{\sigma_{0}}-1\right)^{\delta}
$$

where $\sigma_{0}=3^{1 / 2} k$ denotes the yield stress in simple tension. The relation (48) is equivalent to the Cowper-Symonds-Bodner strain rate law [1].

In the case $\delta=1$, the constitutive equations (47) are equivalent to Freudenthal's relations (4) if we assume $2 \eta=k / \gamma$.

In the case of one-dimensional problems of stress with $\delta=1$, the constitutive equations (47) give Sokolovskii's strain rate law [11].

From Eqs. (10) and (46) we have

$$
J_{2}^{1 / 2}=k\left[1+\left(\left[I_{2}^{P}\right]^{1 / 2} / \gamma\right)^{1 / \delta}\right] .
$$

The dependence of $J_{2}^{1 / 2}$ on $\left(I_{2}^{P}\right)^{1 / 2}$ as given by (49) is plotted in Fig. 3 for various values of $\delta$ and $\gamma$. The particular case of $\delta=1$ is shown in Fig. 4 for different values of $\gamma$.

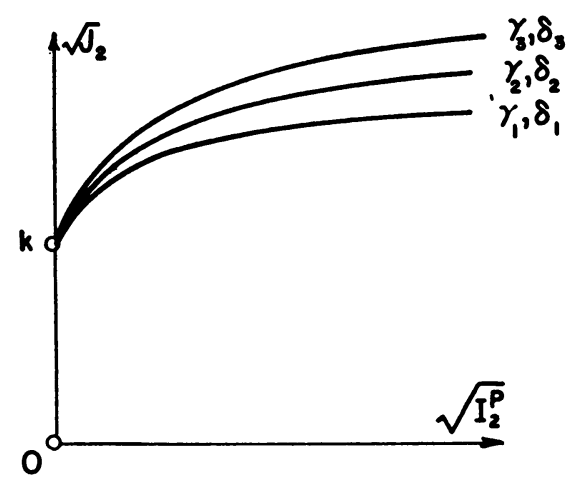

Fra. 3. form

With the use of Eqs. (47), the relaxation equation (25) may now be written in the

$$
J_{2}+4 \mu \gamma\left(\frac{J_{2}^{1 / 2}}{k}-1\right)^{\delta} J_{2}^{1 / 2}=0
$$




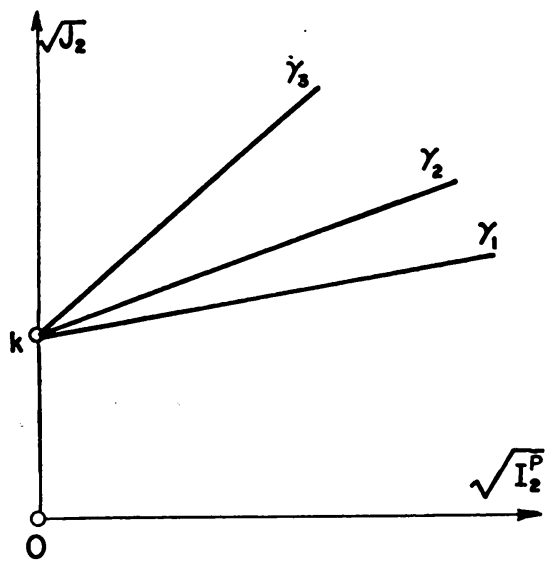

FIG. 4.

The solution of Eq. (50) has form* (30) where

$$
J_{2}^{(n+1)}=J_{2}^{(0)}-4 \mu \gamma \int_{0}^{t}\left(J_{2}^{(n)}\right)^{1 / 2}\left(\frac{\left(J_{2}^{(n)}\right)^{1 / 2}}{k}-1\right)^{\delta} d t
$$

In the special case $\delta=1$, by (50) we have

$$
J_{2}+\frac{4 \mu \gamma}{k}\left(1-\frac{k}{J_{2}^{1 / 2}}\right) J_{2}=0 .
$$

The solution of Eq. (52) (see Freudenthal [5]) may be expressed in closed form as follows:

$$
\left[\frac{J_{2}^{1 / 2}}{k}-1\right]=\left[\frac{\left(J_{2}^{(0)}\right)^{1 / 2}}{k}-1\right] e^{-t / \tau M},
$$

where $\tau_{M}^{-1}=2 \mu \gamma / k$.

As another choice of the function $\Phi(F)$, consider

$$
\Phi(F)=\exp F-1 \text {. }
$$

*By Lagrange's mean value theorem we have

$$
\begin{aligned}
{\left[\left(J_{2}^{\prime}\right)^{1 / 2}\left(\frac{\left(J_{2}^{\prime}\right)^{1 / 2}}{k}-1\right)^{\delta}-\right.} & \left.\left(J_{2}^{\prime \prime}\right)^{1 / 2}\left(\frac{\left(J_{2}^{\prime \prime}\right)^{1 / 2}}{k}-1\right)^{\delta}\right] \\
= & \left(J_{2}^{\prime}-J_{2}^{\prime \prime}\right)\left\{\frac{\partial}{\partial J_{2}}\left[J_{2}^{1 / 2}\left(\frac{J_{2}^{1 / 2}}{k}-1\right)^{\delta}\right]\right\}_{J_{2}=J_{2}^{\prime}+\theta\left(J_{2}^{\prime}-J_{2}^{\prime \prime}\right)}
\end{aligned}
$$

for $0<\theta<1$. Since we can easily show that the derivative

$$
\frac{\partial}{\partial J_{2}}\left[J_{2}^{1 / 2}\left(\frac{J_{2}^{1 / 2}}{k}-1\right)^{\delta}\right]
$$

is bounded, the last result immediately gives the Lipschitz inequality. 
The stress-strain relations (7) can then be written

$$
\begin{array}{ll}
\dot{e_{i j}}=\frac{1}{2 \mu} s_{i i}+\gamma\left\{\exp \left(\frac{J_{2}^{1 / 2}}{k}-1\right)-1\right\} \frac{s_{i j}}{J_{2}^{1 / 2}}, & \text { if } \quad J_{2}^{1 / 2}>k, \\
\dot{e_{i j}}=\frac{1}{2 \mu} \dot{s}_{i j}, & \text { if } J_{2}^{1 / 2} \leq k, \\
\dot{\epsilon_{i i}}=\frac{1}{3 K} \sigma_{i i} . &
\end{array}
$$

For one-dimensional states of stress the relations (55) furnish the strain rate law

$$
\left.\dot{\epsilon}=\frac{\sigma^{\cdot}}{E}+\eta \exp \left(\frac{\sigma}{\sigma_{0}}-1\right)-1\right\}
$$

which, in slightly different form, was introduced by Malvern [7].

From (10) and (54) we have

$$
J_{2}^{1 / 2}=k\left[1+\ln \left(1+\frac{\left(I_{2}^{P}\right)^{1 / 2}}{\gamma}\right)\right] .
$$

The result given by (57) is plotted in Fig. 5, for different values of $\gamma$.

The relaxation equation (25) now has the following form

$$
J_{2}^{\cdot}+4 \mu \gamma\left[\exp \left(\frac{J_{2}^{1 / 2}}{k}-1\right)-1\right] J_{2}^{1 / 2}=0
$$

and its solution is given* by (30) where

$$
J_{2}^{(n+1)}=J_{2}^{(0)}-4 \mu \gamma \int_{0}^{t}\left[\exp \left(\frac{\left(J_{2}^{(n)}\right)^{1 / 2}}{k}-1\right)-1\right]\left(J_{2}^{(n)}\right)^{1 / 2} d t .
$$

Let us assume $\gamma=1$ and introduce the following functions:

$$
\begin{aligned}
\Phi_{\mathrm{I}} & =\sum_{\alpha=1}^{N} A_{\alpha}\left[\exp \left(\frac{J_{2}^{1 / 2}}{k}-1\right)^{\alpha}-1\right], \\
\Phi_{\mathrm{II}} & =\sum_{\alpha=1}^{N} B_{\alpha}\left(\frac{J_{2}^{1 / 2}}{k}-1\right)^{\alpha} .
\end{aligned}
$$

Then the stress-strain relation (7) can be written

$$
\left.\begin{array}{ll}
\dot{e_{i i}}=\frac{1}{2 \mu} s_{i i}+\sum_{\alpha=1}^{N} A_{\alpha}\left[\exp \left(\frac{J_{2}^{1 / 2}}{k}-1\right)^{\alpha}-1\right] \frac{s_{i j}}{J_{2}^{1 / 2}}, & \text { if } \quad J_{2}^{1 / 2}>k, \\
\dot{e_{i i}}=\frac{1}{2 \mu} s_{i i}, & \text { if } J_{2}^{1 / 2} \leq k, \\
\dot{\epsilon_{i i}}=\frac{1}{3 K} \sigma_{i i}, &
\end{array}\right\}
$$

*It can easily be shown that the Lipschitz inequality is satisfied for the function

$$
\left\{J_{2}^{1 / 2}\left[\exp \left(\frac{\dot{J}_{2}^{1 / 2}}{k}-1\right)-1\right]\right\}
$$

(see the footnote on p. 329). 


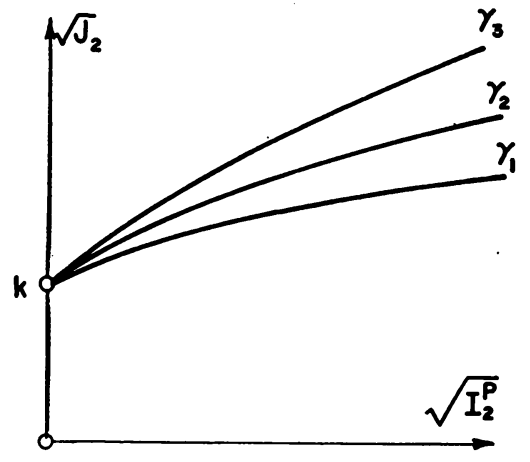

FIG. 5.

or

$$
\begin{array}{ll}
\dot{e_{i i}}=\frac{1}{2 \mu} s_{i i}+\sum_{\alpha=1}^{N} B_{\alpha}\left(\frac{J_{2}^{1 / 2}}{k}-1\right)^{\alpha} \frac{s_{i j}}{J_{2}^{1 / 2}}, & \text { if } J_{2}^{1 / 2}>k, \\
\dot{e_{i j}}=\frac{1}{2 \mu} s_{i i}, & \text { if } J_{2}^{1 / 2} \leq k, \\
\dot{\epsilon_{i i}}=\frac{1}{3 K} \sigma_{i i} . &
\end{array}
$$

It is obvious that all functions $\Phi$ discussed before can be regarded as special cases of (60) or (61).

The solutions of the relaxation equations for the functions (60) and (61) have the form (30) where

$$
\begin{gathered}
J_{2}^{(n+1)}=J_{2}^{(0)}-4 \mu \int_{0}^{t}\left(J_{2}^{(n)}\right)^{1 / 2} \sum_{\alpha=1}^{N} A_{\alpha}\left[\exp \left(\frac{\left(J_{2}^{(n)}\right)^{1 / 2}}{k}-1\right)^{\alpha}-1\right] d t \\
J_{2}^{(n+1)}=J_{2}^{(0)}-4 \mu \int_{0}^{t}\left(J_{2}^{(n)}\right)^{1 / 2} \sum_{\alpha=1}^{N} B_{\alpha}\left(\frac{\left(J_{2}^{(n)}\right)^{1 / 2}}{k}-1\right)^{\alpha} d t
\end{gathered}
$$

respectively.

To determine the constants $A_{\alpha}$ and $B_{\alpha}(\alpha=1,2, \cdots, N)$ we can use the experimental data performed for instance by Clark and Duwez [4]. This problem and the problem of the selection of the constants $\gamma$ and $\delta$ are fully discussed in the work [8].

Acknowledgement. The author is indebted to Professor W. Prager for his critical reading of the manuscript and numerous suggestions for improvements, and to Professors E. H. Lee, P. S. Symonds, and S. R. Bodner for valuable discussions of some parts of this work.

\section{BiBLIOGRAPHY}

1. S. R. Bodner and P. S. Symonds, Plastic deformation in impact and impulsive loading of beams, "Plasticity" (Edited by E. H. Lee and P. S. Symonds), Pergamon Press, New York, 1960, 488-500

2. J. D. Campbell, The yield of mild steel under impact loading, J. Mech. Phys. Solids 3, 54-62 (1954)

3. J. D. Campbell and J. Duby, The yield behavior of mild steel in dynamic compression, Proc. Roy. Soc. 236A, 24-40 (1956)

4. D. S. Clark and P. E. Duwez, The influence of strain rate on some tensile properties of steel, Proc. Amer. Soc. Testing Materials 50, 560-575 (1950) 
5. A. M. Freudenthal, The mathematical theories of the inelastic continuum, Handbuch der Physik VI, Springer-Verlag, Berlin, 1958

6. K. Hohenemser and W. Prager, Über die Ansätze der Mechanik isotroper Kontinua, Zeitschrift f. angew. Math. u. Mech. 12, 216-226 (1932); see also W. Prager, Mécanique des solides isotropes au delà du domaine élastique, Mémorial Sci. Math. 87, Paris, 1937, Eq. (47) on p. 27

7. L. E. Malvern, The propagation of longitudinal waves of plastic deformation in a bar of material exhibiting a strain-rate effect, J. Appl. Mech. 18, 203-208 (1951)

8. P. Perzyna, The study of the dynamical behavior of rate sensitive plastic materials, to be published

9. W. Prager, Linearization in visco-plasticity, Oesterreichisches Ing.-Arch. 15, 152-157 (1961)

10. W. Prager, Introduction to mechanics of continua, Ginn and Company, Boston, 1961

11. V. V. Sokolovskii, Propagation of elastic-visco-plastic waves in bars, (in Russian), Prikl. Mat. Mekh. $12,261-280$ (1948) 